\title{
High-power Vibration Generator Using Gyroscopic Effect
}

\author{
Hiroshi Hosaka, ${ }^{1 *}$ Yoshinori Oonishi, ${ }^{1}$ Yuki Tajima, ${ }^{1}$ and Akira Yamashita ${ }^{2}$ \\ ${ }^{1}$ Department of Human and Engineered Environment, Graduate School of Frontier Sciences, \\ The University of Tokyo, Kashiwa-no-ha, Kashiwa, Chiba 277-8563, Japan \\ ${ }^{2}$ Seigi Mold Co., Ltd., Hiratsuka, Shiroi, Chiba 270-1402, Japan \\ (Received January 14, 2019; accepted March 18, 2019)
}

Keywords: energy harvesting, gyroscope, power generator, vibration

A gyroscopic power generator that generates a power of $1.8 \mathrm{~W}$ is developed with a rotor of $100 \mathrm{~mm}$ diameter and $500 \mathrm{rpm}$ spin speed. In conventional vibration generators, which use simple vibrations of an inner pendulum, the output power has been less than $60 \mathrm{~mW}$. Gyroscopic generators increase the inertial force by rotating the pendulum at a high speed and generate about 50 times greater power than the conventional ones. However, wearable gyro generators that operate under arbitrary vibrations have not been realized because the gyro torque and the electromechanical transformation efficiency are greatly reduced by miniaturization. In this study, first, a theoretical model is developed to clarify the basic characteristics of the generator. Next, a desktop-sized generator that works under any vibration is developed using highly precise motors and gears determined by the theory; the optimum rotor, motor, and gear parameters are decided according to the approximate solution of the mathematical model. Next, mechanical and electrical characteristics are measured to show the validity of the theory. Finally, a wearable test device is produced by modifying a 2.52" hard disk drive (HDD) to show the possibility of obtaining a practical generator.

\section{Introduction}

IoT devices are being realized in many fields. When they are installed in moving objects, energy supply is the largest problem. To solve this, energy harvesting techniques have been studied. ${ }^{(1)}$ Vibration generators have been most intensively studied because vibration exists anywhere and the amount of energy generated is large. An example of a vibration generator is PMG17 from Perpetuum Ltd., ${ }^{(2)}$ which is used for train diagnostics monitoring. The maximum power is $50 \mathrm{~mW}$. A pioneering wearable generator is a wrist watch generator. Its average power is $10 \mu \mathrm{W}$. Since the mechanical power of a human arm is about $10 \mathrm{~W}$ in daily life, the efficiency is only $10^{-6}$. The low efficiency is due to the low inertial force of the vibrating mass. Conventional generators use the inertial force of a simple pendulum as shown in Fig. 1(a). The part generating electricity is modeled as a dashpot. When the generator is excited at the resonant frequency, its average power $P_{l}$ is given by Eq. (1), where $m$ is the mass, $A$ is the input amplitude, $B$ is the relative motion of the mass, and $\omega_{i}$ is the frequency.

\footnotetext{
*Corresponding author: e-mail: hosaka@edu.k.u-tokyo.ac.jp
}

https://doi.org/10.18494/SAM.2019.2296 


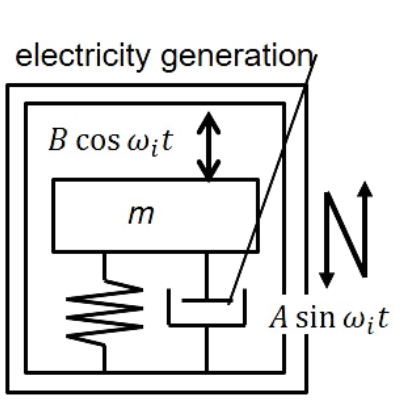

(a)

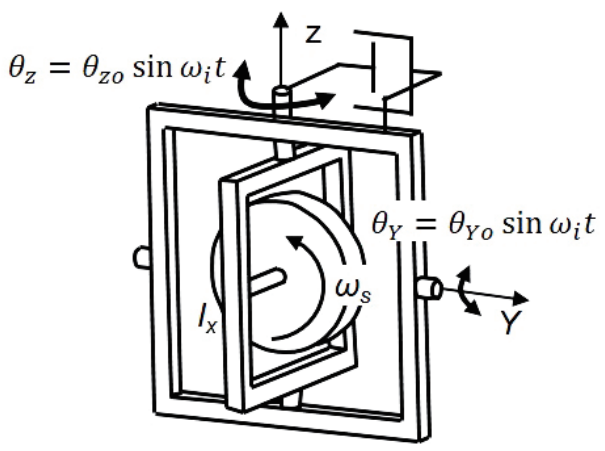

(b)

Fig. 1. (a) Simple pendulum and (b) gyroscopic generators.

$$
P_{l}=\frac{1}{2} m A B \omega_{i}^{3}
$$

To increase the inertial force at low frequencies, generators that use the gyroscopic effect have been studied. They increase the angular momentum of the mass by spinning it at a high speed. In Fig. 1(b), the gyro generator is swung around the $Y$-axis, then the gyro torque generates vibration around the $z$-axis and it moves the part generating electricity. Its average power $P_{o}$ is given by Eq. (2), where $I_{x}$ is the inertia moment, $\omega_{s}$ is the spin speed, $\theta_{Y o}$ is the input amplitude, and $\theta_{z o}$ is the precession amplitude. Its derivation is explained in Sect. 3.4.

$$
P_{o}=\frac{1}{2} I_{x} \theta_{Y o} \theta_{z o} \omega_{i}^{2} \omega_{S}
$$

A case is studied in that the pendulum and rotor have the same size and weight, the input vibration frequency is the same in both generators, and the input and relative vibration amplitudes of the linear generator equal the rotor edge displacements in the input and precession rotations of the gyro generator. Then, Eqs. (3)-(5) stand where $r$ is the rotor radius.

$$
\begin{gathered}
I_{x}=\frac{1}{2} m r^{2} \\
A=\theta_{Y o} r \\
B=\theta_{z o} r
\end{gathered}
$$

The ratio of $P_{o}$ to $P_{l}$ is given as

$$
P_{o} / P_{l}=\omega_{s} / 2 \omega_{i}
$$


In many cases, environmental vibrations have a low frequency, such as those in human movement and the pitting of buoys. On the other hand, the spin speed is easily increased to thousands of rpm such as in hard disk drives (HDDs). If the input frequency is $1 \mathrm{~Hz}$ and the rotation speed is $100 \mathrm{~Hz}$, the power rate is 50 . Thus, the gyro generator is suitable for lowfrequency applications such as wearable devices. Here, the weight of the generator is assumed to be much smaller than that of the vibration source and the movement of the source is not affected by the generator. That is, the input is the displacement or angle, not force nor torque. The expected vibration sources and their weights that satisfy this condition are as follows: human bodies (50 kg), fishing net buoys ( $20 \mathrm{~kg}$ ), navigation buoys $(1 \mathrm{t})$, transportation pallets $(1 \mathrm{t})$, and containers $(20 \mathrm{t})$.

There have been two types of gyro generator, namely, motor- and friction-driven types. The motor-driven type was first invented as an application of gyrostabilizers for ships in 1917. ${ }^{(3)}$ Recently, Bracco et al. ${ }^{(4)}$ have made a prototype of a wave-activated power generator whose rotor weight is $200 \mathrm{~kg}$ and machine length is $4 \mathrm{~m}$. This type of gyro generator has been very large, because the gyro torque, electromagnetic induction, and coil resistance highly depend on the size. Miniaturization greatly reduces the generated power, and even the motor spin power cannot be harvested. The friction-driven type was first invented by Mishler ${ }^{(5)}$ in 1973 and commercialized as a wrist training tool. It rotates a mass through the friction force caused by precession. It produces a power of about $0.1 \mathrm{~W}^{(6,7)}$ However, it can operate only under the vibration that synchronizes the precession cycle.

The purpose of this research is to develop a wearable generator that works under arbitrary vibrations. We focus on the miniaturization of the motor-driven type. First, a basic configuration of the generator is presented. Next, electrical and mechanical equations are derived to obtain guidelines for high performance. Next, a desktop-sized experimental apparatus that generates a power of $1.8 \mathrm{~W}$ is developed. Next, the calculated results are verified by the experiment. Finally, a test device of a wearable generator is produced by modifying a PC-embedded HDD to obtain a prospect of practical generators.

\section{Nomenclature}

$C_{m}$ : electromagnetic damping coefficient of generating motor $\tau_{m} / \omega_{m}$ when output terminals are short-circuited

$\vec{e}$ : basis vector

$\vec{h}$ : rotor angular momentum

$\vec{I}$ : vector whose elements are principal moments of rotor inertia

$I_{m}$ : electric current of generating motor

$K_{t}$ : torque constant

$k_{s}$ : spring constant

$N$ : gear ratio

$o$ : suffix of amplitude

$P$ : generated power

$R_{m}$ : motor resistance 
$R_{o}$ : load resistance

$V_{m}$ : voltage of generating motor

$x$ : rotor spin axis

$x^{\prime}$ : normal axis to outer gimbal surface

$X$ : normal axis to fixed frame surface

$y$ : normal axis to inner gimbal surface

$Y$ : rotation axis of outer gimbal

$z$ : rotation axis of inner gimbal

$Z$ : vertical axis

$\zeta$ : damping ratio

$\eta$ : gear efficiency

$\theta$ : angle

$\vec{\tau}$ : torque vector

$\tau_{g}$ : gear input torque

$\tau_{m}$ : torque of generating motor

$\tau_{z}:$ rotor precession torque

$\vec{\omega}$ : rotor angular velocity

$\omega_{i}$ : exciting angular frequency

$\omega_{m}$ : angular velocity of generating motor

$\omega_{s}$ : rotor spin speed

$\vec{\Omega}$ : angular velocity of inner gimbal

If $X, Y, Z, x^{\prime}, x, y, z$, and $o$ are used as suffixes of $I, \theta, \omega$, and $\vec{e}$, they show the directional components of the parameters or corresponding vectors.

\section{Configuration and Theory}

\subsection{Configuration}

The configuration of our target generator is shown in Fig. 2. The generator is made of a rotor, a spindle motor, a secondary battery, acceleration gears, a generating motor, a precession

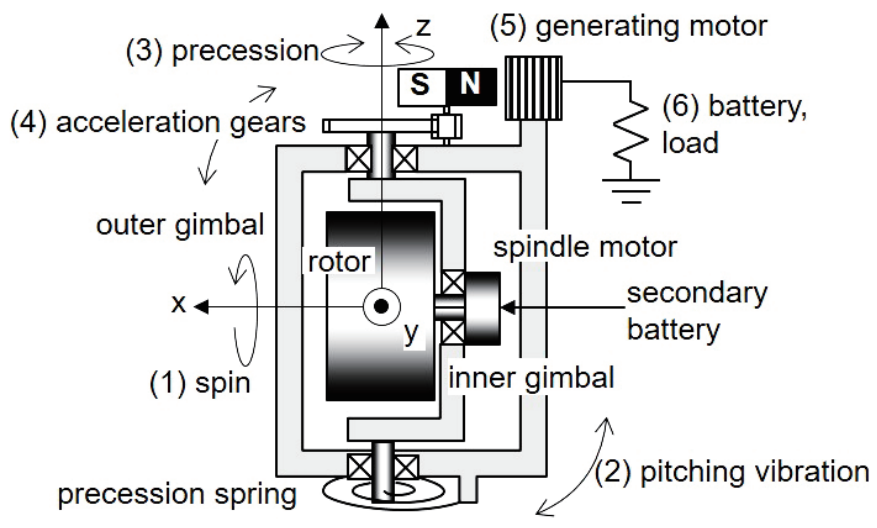

Fig. 2. Configuration of gyro generator. 
spring, and outer and inner gimbals. There are two motors in the generator. One motor rotates the rotor by the secondary battery or an external electrical source. We call it a "spindle motor". The other motor is used as a generator driven by the acceleration gears and generates electricity. We call it a "generating motor". The operation sequence is as follows. (1) A rotor is rotated by a spindle motor driven by a battery. (2) A pitching vibration is applied by an external torque. (3) The rotor begins precession due to the gyro effect. (4) The precession speed is accelerated by the gear. (5) An electric power is generated by the generating motor. (6) The battery is charged and the excess power is supplied to a load. If the power consumed by the spindle motor is smaller than that generated by the generating motor, this device works as a generator.

\subsection{Dynamics of rotor}

A mathematical model of the generator is constructed and its performance is studied. We assume that the spin speed and pitching vibration are constant. First, the equation of the rotor motion is derived. To show the three-dimensional rotation of the rotor, we use the three coordinates shown in Fig. 3. The rotor is connected to the ground through the inner and outer gimbals and a fixed frame. The coordinate system fixed to the frame is called the $X Y Z$ system, where $Z$ is the vertical direction, $Y$ is the rotating axis of the outer gimbal, and $X$ is the direction perpendicular to the frame plane. The coordinate system fixed to the outer gimbal is called the $x^{\prime} Y z$ system, where $z$ is the rotating axis of the inner gimbal and $x^{\prime}$ is perpendicular to the outer gimbal surface. The coordinate system fixed to the inner gimbal is called the $x y z$ system, where $x$ is the rotor spin axis and $y$ is the radial direction of the rotor. The origin of all coordinates is the center of the rotor. The pitching angle is denoted as $\theta_{Y}$, the precession angle as $\theta_{z}$, their angular velocities as $\omega_{Y}$ and $\omega_{z}$, and the rotor spin speed as $\omega_{s}$. Note that $\omega_{s}$ is a relative velocity measured from the inner gimbal, whereas $\omega_{Y}$ and $\omega_{z}$ are the absolute velocities measured from $X Y Z$ coordinates. The absolute velocity around the $x$-axis, $\omega_{x}$, is $\omega_{s}$ plus the inner gimbal velocity $\omega_{Y} \sin \theta_{z}$.

The rotation of the rotor is given by the law of angular momentum, Eq. (7), where $d / d t$ is the time derivative in the $X Y Z$ frame.

$$
\vec{\tau}=d \vec{h} / d t
$$
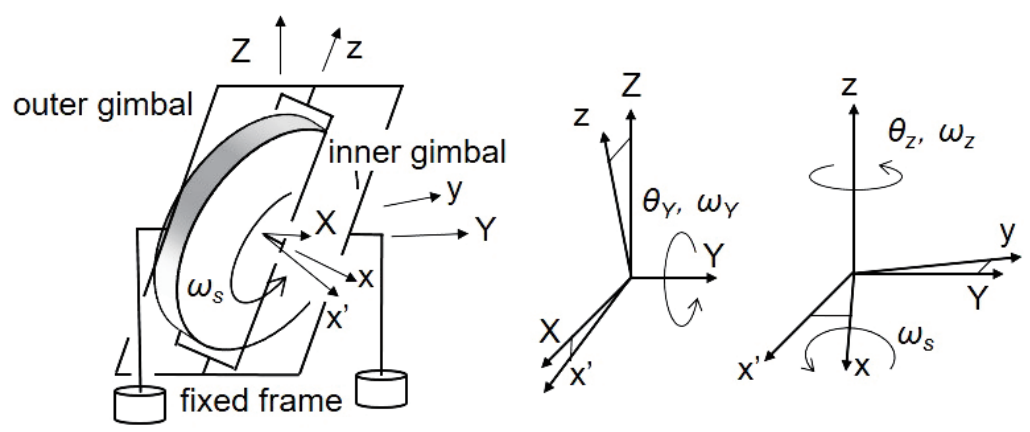

Fig. 3. Coordinate systems of rotor and gimbals. 
The angular momentum $\vec{h}$ is decomposed into the principal directions of the rotor, namely, $x, y$, and $z$, as follows.

$$
\vec{h}=I_{x} \omega_{x} \overrightarrow{e_{x}}+I_{y} \omega_{y} \overrightarrow{e_{y}}+I_{z} \omega_{z} \overrightarrow{e_{z}}
$$

Also, we decompose the time derivative of the fixed coordinates $d / d t$ to that of the rotating coordinates $x y z, \partial / \partial t$, and the coordinate rotation $\vec{\Omega}$. The symbol $\times$ shows the vector product.

$$
d / d t=\partial / \partial t+\vec{\Omega} \times
$$

The angular velocity $\vec{\Omega}$ is the sum of the input angular velocity $\omega_{Y}$ and the precession angular velocity $\omega_{z}$. The angular velocity $\vec{\omega}$ is the sum of $\vec{\Omega}$ and the rotor spin $\omega_{s}$. They are given as follows.

$$
\begin{gathered}
\vec{\Omega}=\omega_{Y} \overrightarrow{e_{Y}}+\omega_{z} \overrightarrow{e_{z}} \\
\vec{\omega}=\omega_{Y} \overrightarrow{e_{Y}}+\omega_{z} \overrightarrow{e_{z}}+\omega_{s} \overrightarrow{e_{x}}
\end{gathered}
$$

There is a relationship among basis vectors as follows.

$$
\overrightarrow{e_{Y}}=\overrightarrow{e_{x}} \sin \theta_{z}+\overrightarrow{e_{y}} \cos \theta_{z}
$$

By inserting Eq. (12) into Eqs. (10) and (11) and eliminating $\overrightarrow{e_{Y}}$, and by applying Eqs. (8)-(10) to Eq. (7), we obtain the following equation, where the third term on the right-hand side is the gyro effect.

$$
\tau_{z}=I_{z} \ddot{\theta}_{z}+\left(I_{y}-I_{x}\right) \omega_{Y}^{2} \sin \theta_{z} \cos \theta_{z}-I_{x} \omega_{Y} \omega_{s} \cos \theta_{z}
$$

The pitching angle is given as a sinusoidal wave of amplitude $\theta_{Y o}$ and angular frequency $\omega_{i}$.

$$
\theta_{Y}=\theta_{Y o} \sin \omega_{i} t
$$

\subsection{Equations of other components}

The generating motor has an inductance $L_{m}$. However, $L_{m}$ is less than $1 \mathrm{mH}$ and the resistance $R_{m}$ is larger than $1 \Omega$; thus, the time constant $L_{m} / R_{m}$ is less than $1 \mathrm{~ms}$, which is much smaller than the vibration period of $1 \mathrm{~s}$. Therefore, the inductance is neglected. Then, the equations for other components are linear algebraic equations, which are shown below. 
Spring

$$
\tau_{z}+k_{s} \theta_{z}+\tau_{g}=0
$$

Gear

$$
\begin{gathered}
\omega_{m}=N \omega_{g} \\
\tau_{m}=\eta \tau_{g} / N
\end{gathered}
$$

Generating motor

$$
\begin{gathered}
V_{m}=K_{t} \omega_{m} \\
\tau_{m}=K_{t} I_{m}
\end{gathered}
$$

Load resistance

$$
\begin{gathered}
V_{m}=\left(R_{m}+R_{o}\right) I_{m} \\
P=I_{m}{ }^{2} R_{o}
\end{gathered}
$$

\subsection{Approximate solution}

An approximate solution is obtained in a closed form if the conditions $\omega_{z} \ll \omega_{S}$ and $\theta_{z} \ll 1$ stand. Then, the second term of Eq. (13) is neglected, $\cos \theta_{z}$ is approximated as 1 , and the equations are combined to an equation of a forced vibration of a linear, single-degree-offreedom system.

$$
I_{z} \ddot{\theta}_{z}+\frac{N^{2} K_{t}^{2}}{\eta\left(R_{m}+R_{o}\right)} \dot{\theta}_{z}+k_{s} \theta_{z}=I_{x} \omega_{s} \theta_{Y_{O}} \omega_{i} \cos \omega_{i} t
$$

At the steady state, $\theta_{z o}$ and $P_{o}$, which are the amplitudes of $\theta_{z}$ and $P$, are respectively given as

$$
\begin{gathered}
\theta_{z o}=\frac{I_{x} \omega_{s} \theta_{Y o} \omega_{i}}{\sqrt{\left(k_{s}-I_{z} \omega_{i}{ }^{2}\right)^{2}+\frac{N^{4} C_{m}{ }^{2} \omega_{i}^{2}}{\eta^{2}\left(1+R_{o} / R_{m}\right)^{2}}}}, \\
P_{o}=\frac{R_{o} / R_{m}}{2\left(1+R_{o} / R_{m}\right)^{2}} N^{2} C_{m} \theta_{z o}{ }^{2} \omega_{i}{ }^{2},
\end{gathered}
$$


where $C_{m}=K_{t}^{2} / R_{m}$ is the electromagnetic damping coefficient of the generating motor when its electrical terminals are short-circuited.

If the input frequency $\omega_{i}$ is the resonant frequency of the precession vibration $\omega_{i}=\sqrt{k_{s} / I_{z}}$, and the mechanical and electrical losses are neglected as $\eta=1$ and $R_{m}=0, P_{o}$ becomes

$$
P_{o}=\frac{1}{2} I_{x} \omega_{S} \omega_{Y o} \omega_{z o}
$$

Equation (1) is obtained by using the relations $\omega_{Y o}=\theta_{Y o} \omega_{i}$ and $\omega_{z o}=\theta_{z o} \omega_{i}$.

\subsection{Effect of precession spring}

By solving the nonlinear equations, we examine the effect of the precession spring. Figure 4(a) shows the precession angle without a spring. The parameters are as follows: $\theta_{Y_{o}}=20 \mathrm{deg}, \omega_{i}=2.5 \mathrm{~Hz}, \omega_{s}=500 \mathrm{rpm}, I_{x}=5.3 \mathrm{gm}^{2}, I_{z}=2.97 \mathrm{gm}^{2}, N=105, \eta=0.61$, $K_{t}=21.4 \mathrm{mNm} / \mathrm{A}, R_{m}=28 \Omega$, and $R_{o}=30 \Omega$. These are almost the same as those of the experimental apparatus in Sect. 4.1. The center of the vibration shifts gradually upward and the vibration finally stops at $90 \mathrm{deg}$. This occurs owing to the dependence of the gyro torque on the precession angle. The gyro torque in Eq. (13) includes $\cos \theta_{z}$. Thus, the shifting torque from $\theta_{z}=0$ is larger than the returning torque to 0 . Thus, the vibration center moves from 0 to the edge. When the center of the vibration is $90 \mathrm{deg}$, the torque is nearly 0 and the precession vibration stalls. This means that, when there is no spring, a stable power generation is impossible.

Figure 4(b) shows the precession angle in the case with a spring. In this case, the vibration center does not shift and the amplitude remains constant. This occurs because the restoring torque of the spring is larger than the shifting torque. This shows that an appropriate spring makes a stable power generation possible.

\subsection{Frequency characteristics}

By using the approximate solutions Eqs. (23) and (24), we study the relationships among the generated power, input frequency, and gear ratio. To guarantee the validity of linearity, the

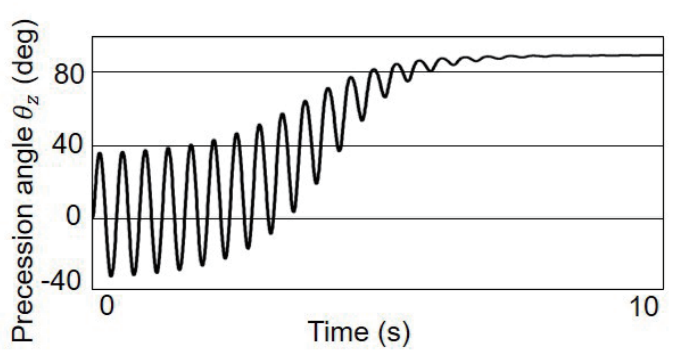

(a)

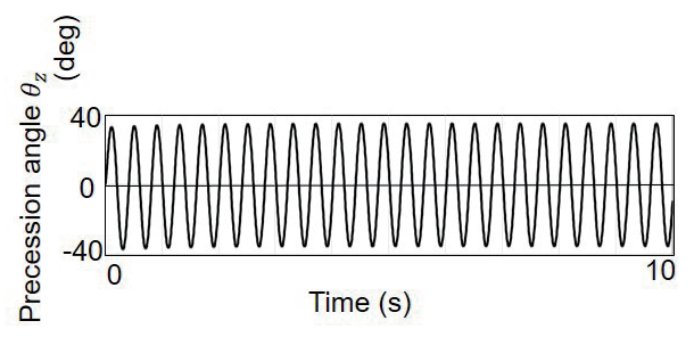

(b)

Fig. 4. Precession angles obtained with and without spring. (a) $k_{s}=0$. (b) $k_{s}=3.84 \mathrm{mNm} / \mathrm{deg}$. 
input angle is set to a value as small as $1 \mathrm{deg}$. The other calculation conditions are as follows: $\omega_{s}=100 \mathrm{rpm}, I_{x}=1.12 \mathrm{gm}^{2}, I_{z}=0.584 \mathrm{gm}^{2}, \eta=0.61, K_{t}=21.4 \mathrm{mNm} / \mathrm{A}, R_{m}=30 \Omega, R_{o}=30 \Omega$, and $k_{s}=1.92 \mathrm{mNm} / \mathrm{deg}$. The results are shown in Fig. 5. Since the system is equivalent to a forced vibration system, the power curve has a peak at the resonant frequency of $2.2 \mathrm{~Hz}$. The reason why the peak becomes sharper as the gear ratio decreases is that the electromagnetic force works as a damping force and is proportional to $N^{2}$, as shown in the second term of Eq. (22). This means that, with a high gear ratio, power generation becomes insensitive to the input frequency and advantageous for varying-frequency vibration.

\subsection{Design guidelines}

On the basis of the simplified solutions Eqs. (23) and (24), design guidelines are examined. From Eq. (24), $P_{o}$ becomes maximum at $R_{o}=R_{m}$. Also, $P_{o}$ increases with $N$ and $C_{m}$. Since the generating motor characteristics appear only as $C_{m}$ and $R_{o} / R_{m}$ in Eqs. (23) and (24), $C_{m}$ is the only parameter related to the motor performance at $R_{o}=R_{m} . C_{m}$ increases with the magnet volume, the number of coil turns, and the coil wire thickness. Since these increase the motor volume, the upper limit of $C_{m}$ is determined by the allowable motor space. The motor with large $C_{m}$ and small volume is necessary for high-power generators. The maximum $N$ is limited by the gear strength. If $N$ is very large, the friction increases and the first gear is broken. Also, $P_{o}$ increases with $\theta_{o}$. Equation (24) shows that $\theta_{o}$ increases with $I_{x}$ and $\omega_{s}$. $I_{x}$ should be increased as far as the device size and bearing strength allow. $\omega_{s}$ is limited by the friction of the rotor. Usually, the bearing friction power is proportional to 1 to 2 powers of $\omega_{s}$, whereas the power generated by the gyro is proportional to $\omega_{s}$ if $\theta_{Y o}$ and $\theta_{z o}$ are constant. Thus, there exists a $\omega_{s}$ that maximizes the net output power. The optimum $\omega_{s}$ depends on the gyro torque and friction and electrical losses.

\section{Experiment}

\subsection{Experimental apparatus}

To verify the mathematical model of the generator shown in Sects. 3.2 and 3.3, the experimental apparatus shown in Fig. 6 is developed. The rotor spins around a horizontal shaft. The input vibration is given to the other horizontal shaft manually with the handle.

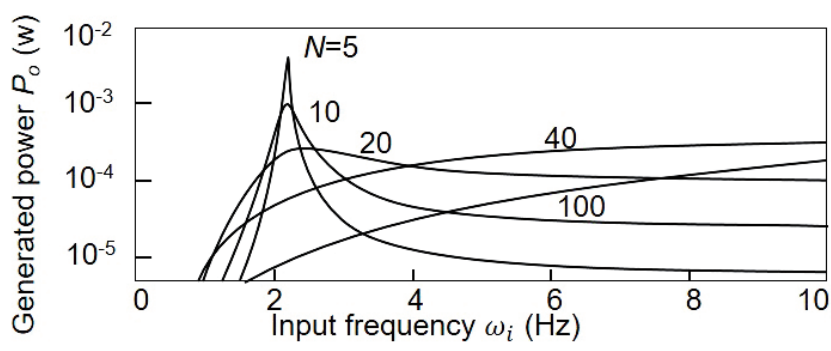

Fig. 5. Effects of excitation frequency and gear ratio on generated power. 


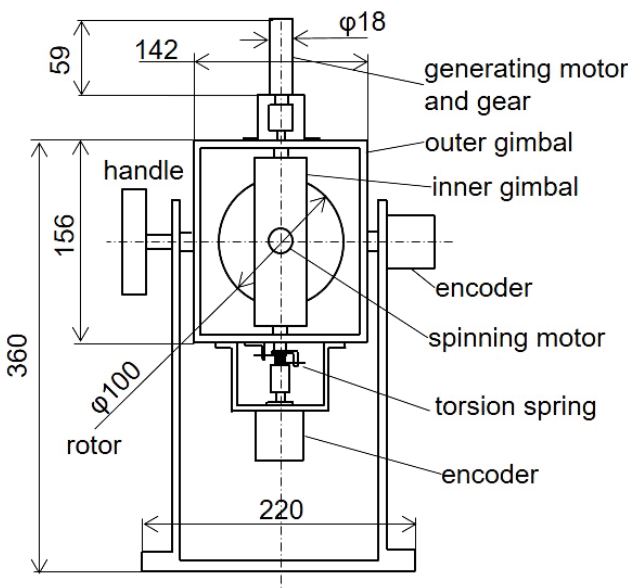

(a)

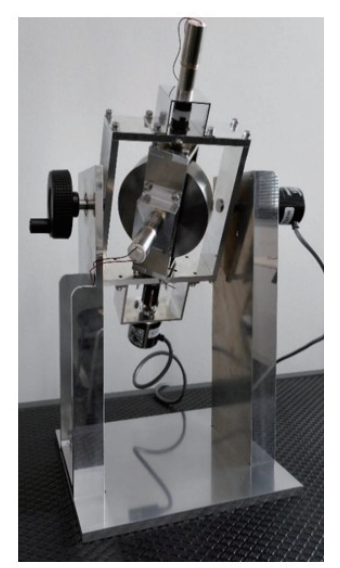

(b)

Fig. 6. (Color online) (a) Dimension and (b) photoglaph of experimental apparatus.

The precession is a rotation around the vertical axis. It is transformed into electricity by a generating motor and a gear. The input and precession angles are measured by encoders. The rotor spin speed is measured by a photoreflector attached to the hidden side of the inner gimbal. The rotor, generating motor, and acceleration gear are the key parts. They are selected through the design guidelines. The rotor is $100 \mathrm{~mm}$ in diameter and $30 \mathrm{~mm}$ in thickness, which are the largest dimensions that can be fixed to a human body. The rotor material is tungsten alloy with a specific weight of 18 , which is the heaviest metal capable of precise machining. The generating motor is SCL18 from Adamant Namiki Precision. It has a diameter of $18 \mathrm{~mm}$, a length of $35 \mathrm{~mm}$, a torque constant of $K_{t}=21.4 \mathrm{mNm} / \mathrm{A}$, and a resistance of $R_{m}=28 \Omega . C_{m}$ is $16.33 \mu \mathrm{Nms} / \mathrm{rad}$, which is the largest value observed in the same size motors. The gear has the ratio $N=105$, which is the largest value observed among the acceleration gears of this size. The spin speed $\omega_{s}$ is set to $500 \mathrm{rpm}$, which is decided experimentally to make the net generating power maximum.

\subsection{Comparison of experimental and calculated results}

Figure 7 shows the experimental and calculated results of input and precession angles. The calculated and experimental precession angles are 40 and $31 \mathrm{deg}$, respectively, which are almost in agreement with each other. The reason that the experimental angles are not precisely sinusoidal is that the input vibration is obtained manually.

Figure 8 shows the input angle and generated power. The average power in the calculation is $1.8 \mathrm{~W}$ and that in the experiment is $1.87 \mathrm{~W}$. These values show good agreement. In the experiment, the input electric power necessary to drive the spinning motor is $84.6 \mathrm{~mW}$, which is much smaller than the output power. The net generated power is $1.8 \mathrm{~W}$.

Figure 9 shows the experimental and calculated average powers obtained when the spring constant and spin speed are varied. The symbols show the experimental values and the lines show the calculated values, which show good agreement. $P_{o}$ increases as $\omega_{s}$ increases and $k_{s}$ decreases. This is because $\omega_{s}$ increases the gyro torque and $k_{s}$ decreases the precession angle. 


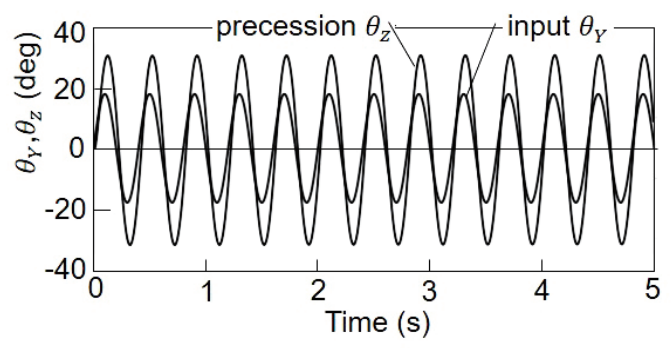

(a)

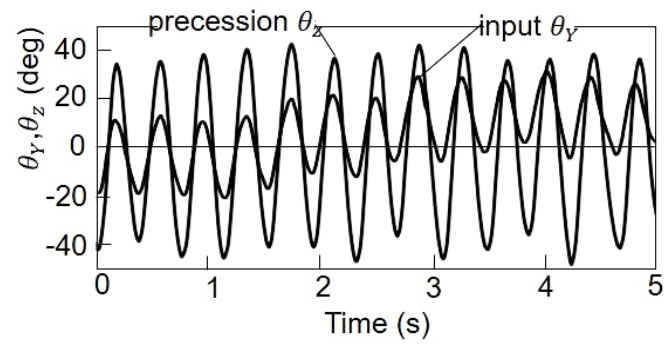

(b)

Fig. 7. Input and precession angles. (a) Calculation. (b) Experiment.

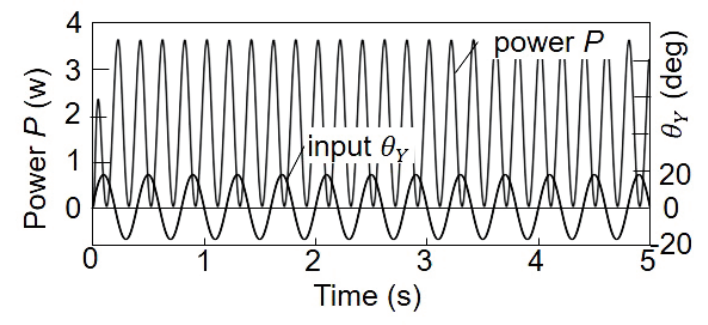

(a)

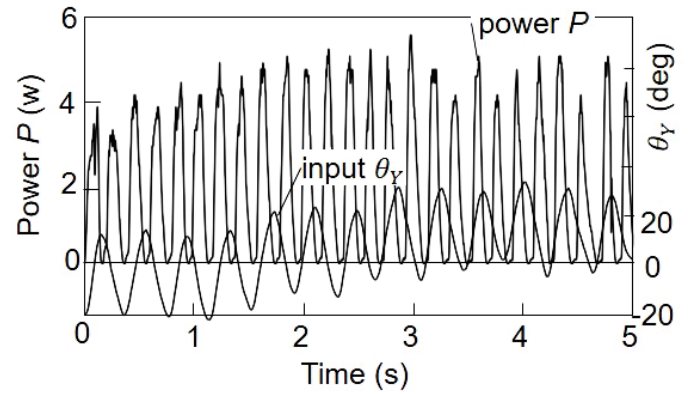

(b)

Fig. 8. Input angle and generated power. (a) Calculation. (b) Experiment.

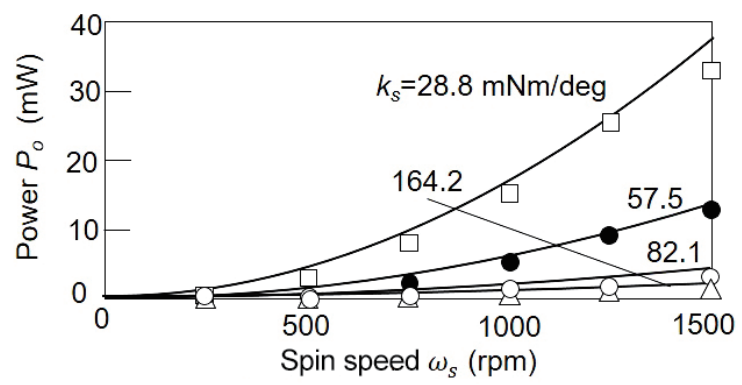

Fig. 9. Generated powers for various spring constants.

Figure 10 shows experimental and calculated average powers obtained using various rotors and springs at various $\omega_{S}$ and $\theta_{Y o}$ as shown in Table 1 . The horizontal axis shows the experimental value and the vertical axis shows the calculated value. The diagonal straight line shows the coincidence of the two values. The results show good agreement.

The output powers of the conventional generators are compared with that of our generator. Since there is no generator with the same size as ours, the power is converted using Eq. (1). The volume of our apparatus is assumed to be that inside the outer gimbal. The vibration distance in the linear generator is the same as the displacement of the gimbal edge. Then, the average powers in Refs. 8 and 9 are both approximately $60 \mathrm{~mW}$. Thus, the average power of our apparatus is 30 times higher than these values. 


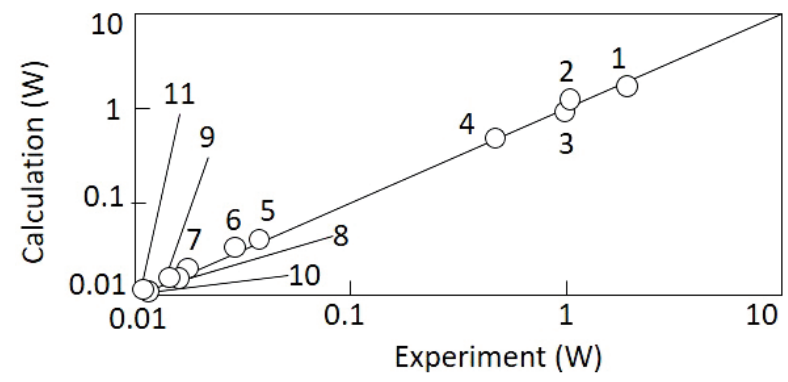

Fig. 10. Experimental and calculated average powers.
Table 1

Experimental and calculated conditions of Fig. 10.

\begin{tabular}{ccccc}
\hline Point & Rotor & Spring & $\omega_{S}(\mathrm{rpm})$ & $\theta_{Y_{o}}(\mathrm{deg})$ \\
\hline 1 & $\mathrm{R} 1$ & $k_{1}$ & 500 & 18 \\
2 & $\mathrm{R} 1$ & $k_{1}$ & 500 & 15 \\
3 & $\mathrm{R} 1$ & $k_{1}$ & 375 & 17 \\
4 & $\mathrm{R} 1$ & $k_{1}$ & 250 & 18 \\
5 & $\mathrm{R} 2$ & $k_{2}$ & 1500 & 25 \\
6 & $\mathrm{R} 2$ & $k_{2}$ & 1250 & 27 \\
7 & $\mathrm{R} 2$ & $k_{2}$ & 1000 & 26 \\
8 & $\mathrm{R} 2$ & $k_{3}$ & 1500 & 27 \\
9 & $\mathrm{R} 2$ & $k_{4}$ & 1500 & 29 \\
10 & $\mathrm{R} 2$ & $k_{3}$ & 1250 & 28 \\
11 & $\mathrm{R} 2$ & $k_{4}$ & 1250 & 30 \\
\hline R1 $\left(\mathrm{gm}^{2}\right): I_{x}=5.3, I_{z}=2.97, \mathrm{R} 2: I_{x}=0.90, I_{z}=0.45$. \\
spring $k_{s}(\mathrm{mNm} / \mathrm{deg}):$ \\
$=60.9$
\end{tabular}

\section{Toward a Practical Generator}

A small device is produced to clarify the problems encountered in realizing a practical generator. The experimental apparatus in Sect. 4.1 is very large because of the spindle motor that protrudes from the rotor surface. Since the inner part of the flywheel does not contribute to the inertia moment, if a spindle motor is embedded in the flywheel, the outer shape of the device will be reduced. A wearable consumer product with an embedded motor is an HDD. A test device is fabricated from a 2.5" HDD by combining a gimbal and a generating motor. A picture is shown in Fig. 11. Its weight is $238 \mathrm{~g}$ and its outer size without the generating motor is $80 \times 90 \times 15 \mathrm{~mm}^{3}$. The spindle part is a 2.5 " HDD. The access arm is removed and the chassis is cut to a square shape. The platter is changed to a stainless disk of $5 \mathrm{~mm}$ thickness. The motor is driven by a commercial sensorless controller chip. The gimbal is made of an aluminum frame, ball bearings, and shafts. The generating motor is the same as that in the experimental apparatus.

The experimental conditions are as follows: $\omega_{s}=3000 \mathrm{rpm}, \omega_{i}=3.5 \mathrm{~Hz}, \theta_{Y o}=16 \mathrm{deg}, \theta_{z o}=$ $5 \mathrm{deg}$, and $R_{o}=26.5 \Omega$. The measured output power is shown in Fig. 12. The average power is $45 \mathrm{~mW}$, whereas the average driving power of the spindle motor is $360 \mathrm{~mW}$. Since the power consumed is 8 times higher than the output power, this device does not work as a generator. The reason for the low power generation is that the spindle shaft of the HDD is supported on one side by a fluid dynamic bearing. Thus, the supporting torque of the bearing is low and the rotor mass and input vibration amplitude cannot be increased. If the shaft is supported by ball bearings on both sides, a heavier rotor is used and larger precession and pitching torques are applied. If the input angle is set to $20 \mathrm{deg}$, the precession angle is set to $30 \mathrm{deg}$, and the rotor material is tungsten, which are the same as those in the apparatus in Sect. 4.1, the generated power becomes $890 \mathrm{~mW}$, which is 16.5 times larger than the current one. This is also much larger than the loss power. This makes us expect the realization of a $530 \mathrm{~mW} 2.5$ " generator. 


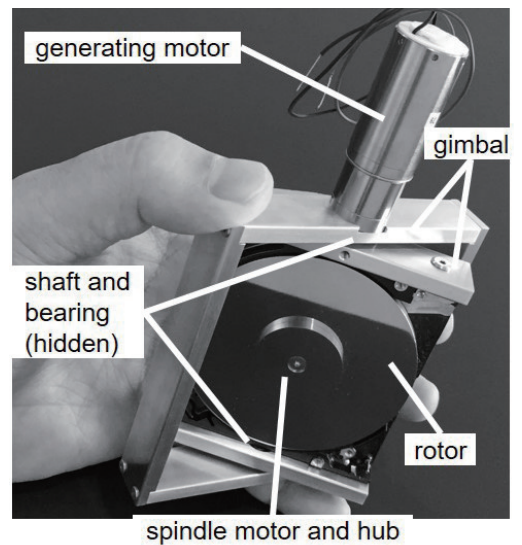

Fig. 11. 2.5" test device.

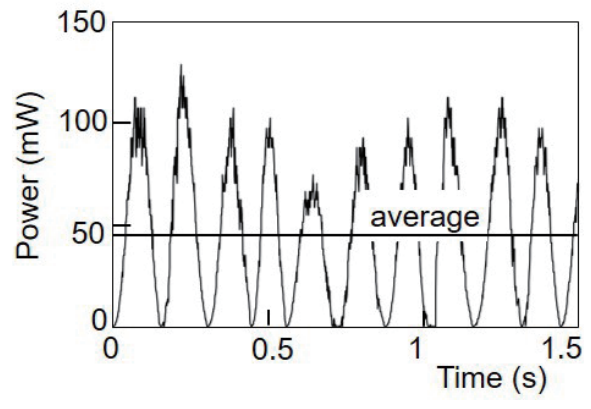

Fig. 12. Output power of $2.5^{\prime \prime}$ generator.

\section{Conclusions}

To realize a small and high-power vibrational generator, test devices using the gyro effect were developed. The conclusions are as follows.

(1) An experimental apparatus was developed with a rotor of $100 \mathrm{~mm}$ diameter and $500 \mathrm{rpm}$ spin speed. By applying a vibration of 17.7 deg amplitude and $2.5 \mathrm{~Hz}$ frequency, we obtained a net power of $1.8 \mathrm{~W}$.

(2) A mathematical model was developed by combining the equations of motion of the rotor and the electrical and mechanical characteristics of a spring, gears, a motor, and a resistance. It was found that a precession spring is necessary for a stable power generation and, by increasing the precession gear ratio, a constant power generation is possible in a wide frequency range.

(3) Power generating characteristics were measured by changing the rotor size, spin speed, and spring constant of the apparatus. The results are in good agreement with the calculated ones.

(4) By modifying a 2.5" HDD, we produced a wearable generator. It generated a power of 45 $\mathrm{mW}$ but consumed $360 \mathrm{~mW}$ for motor spin. It is expected that the generated power will increase to $890 \mathrm{~mW}$ if the stiffness of the spindle shaft is increased by supporting the shaft on both sides.

The gyro generator is related to sensors in two points. First, the major application of the generator is an energy source of sensors mounted on moving objects. Examples are GPS, accelerometers, electrocardiographs, thermometers, salinity sensors and flow velocimeters mounted on human bodies, marine buoys, and transportation equipment. Second, the energy creation principle of our generator is the same as the measurement principle of the gyro sensor. Many of the energy harvesters are varieties of sensors; solar batteries, and wind power and piezoelectric generators correspond to photo, flow, and acceleration sensors. The same techniques are used for the power and sensitivity enhancements. In the gyroscopes, inertia moment, spin speed, electromechanical transformation, and friction are key factors for both power and sensitivity improvements. Our results will be useful in the gyro sensor design. 


\section{Acknowledgments}

This research is supported by the A-STEP Program from the Japan Science and Technology Agency, JST.

\section{References}

1 S. Roundy, P. K. Wright, and J. M. Rabaey: Energy Scavenging for Wireless Sensor Networks (Kluwer Academic Publications, Norwell, M.A., 2004). ISBN-10:1402076630

2 L. Hayes: IEEE Control Syst. Mag. 28 (2008) 107. https://doi.org/10.1109/MCS.2007.911267

3 C. Norden: U. S. Patent 1,236,204 (1917).

4 G. Bracco, A. Cagninei, E. Giorcelli, G. Mattiazzo, D. Poggi, and M. Raffero: Ocean Eng. 120 (2016) 40. https://doi.org/10.1016/j.oceaneng.2016.05.006

5 A. L. Mishler: U. S. Patent 3,726,146 (1973).

6 T. Ishii, J. Iwasaki, and H. Hosaka: Int. J. Appl. Electromagnet. Mech. 36 (2011) 131. https://doi.org/10.3233/ JAE-2011-1351

7 Engadget: Manual Power, https://japanese.engadget.com/2006/01/27/manual-power/ (accessed January 12, 2019).

8 Star Micronics Co., Ltd.: New power generators harness energy from vibrations, https://www.star-m.jp/eng/ topics/3267/ (accessed January 12, 2019).

9 Y. Naruse, N. Matsubara, K. Mabuchi, M. Izumi, and S. Suzuki: J. Micromech. Microeng. 19 (2009) 1. https:// doi.org/10.1088/0960-1317/19/9/094002 